\title{
Downstream Super-magnetosonic Plasma Jet Generation as a Direct Consequence of Shock Reformation
}

Savvas Raptis ( $\sim$ savvra@kth.se)

KTH Royal Institute of Technology https://orcid.org/0000-0002-4381-3197

Tomas Karlsson

KTH Royal Institute of Technology

Andris Vaivads

KTH Royal Institute of Technology https://orcid.org/0000-0003-1654-841X

Craig Pollock

Denali Scientific

Ferdinand Plaschke

Space Research Institute https://orcid.org/0000-0002-5104-6282

Andreas Johlander

University of Helsinki

Henriette Trollvik

KTH Royal Institute of Technology

P.-A. Lindqvist

Royal Institute of Technology https://orcid.org/0000-0001-5617-9765

\section{Article}

Keywords: Earth's bow shock, Magnetospheric Multiscale, super-magnetosonic downstream flows

Posted Date: September 3rd, 2021

DOl: https://doi.org/10.21203/rs.3.rs-711807/v1

License: (c) (i) This work is licensed under a Creative Commons Attribution 4.0 International License.

Read Full License

Version of Record: A version of this preprint was published at Nature Communications on February 1 st, 2022. See the published version at https://doi.org/10.1038/s41467-022-28110-4. 
This preprint is under consideration at Nature Communications. A preprint is a preliminary version of a manuscript that has not completed peer review at a journal. Research Square does not conduct peer review prior to posting preprints. The posting of a preprint on this server should not be interpreted as an endorsement of its validity or suitability for dissemination as established information or for guiding clinical practice.

\title{
1 Downstream Super-magnetosonic Plasma Jet Generation as a Direct Consequence of Shock Reformation
}

\author{
Savvas Rapitis ${ }^{1 *}$, Tomas Karlsson ${ }^{1}$, Andris Vaivads ${ }^{1}$, Craig Pollock ${ }^{2}$, Ferdinand \\ Plaschke $^{3}$, Andreas Johlander ${ }^{4,5}$, Henriette Trollvik ${ }^{1}$, Per-Arne Lindqvist ${ }^{1}$ \\ 1 Division of Space and Plasma Physics - KTH Royal Institute of Technology, Stockholm, Sweden \\ 2 Denali Scientific, Fairbanks, AK, 99709, USA \\ 3 Space Research Institute, Austrian Academy of Sciences, Graz, Austria \\ 4 Department of Physics, University of Helsinki, Finland \\ ${ }^{5}$ Swedish Istitute of Space Physics, Uppsala, Sweden
}

10 Earth's bow shock, resulting from the interac11 tion of the super-magnetosonic solar wind and 12 Earth's magnetic field, has been studied for over ${ }_{13} 50$ years and serves as an ideal astrophysical lab${ }_{14}$ oratory to study collisionless shocks [1, 2]. The ${ }_{15}$ Earth's bow shock offers a unique opportunity to ${ }_{16}$ study it through in-situ measurements. Shocks 17 are one of nature's most powerful particle ac18 celerators and have been connected to relativis19 tic electron acceleration and cosmic rays $[3,4]$. ${ }_{20}$ Upstream shock observations include wave gen${ }_{21}$ eration, wave-particle interactions and SLAMS, 22 while at the shock and downstream, particle ac${ }_{23}$ celeration, magnetic reconnection and plasma jets ${ }_{24}$ can be observed [5-7]. Here, using Magneto${ }_{25}$ spheric Multiscale (MMS) we show the first in${ }_{26}$ situ evidence of super-magnetosonic downstream ${ }_{27}$ flows (jets) generated at the Earth's bow shock as ${ }_{28}$ a direct consequence of shock reformation. Jets ${ }_{29}$ are observed downstream due to a combined ef30 fect of upstream plasma wave evolution and an 31 ongoing reformation cycle of the bow shock. This 32 generation process can also be applicable to plan${ }_{3}$ etary and astrophysical plasmas where collision${ }_{34}$ less shocks are commonly found $[2,3,8,9]$.

35 The type of bow shock that is most challenging to 36 study is the so called "quasi-parallel" shock, where the 37 upstream magnetic field is approximately parallel to the 38 shock's surface normal [5]. Downstream of it, the shocked 39 solar wind form a highly variable environment named the 40 magnetosheath. The shock and its upstream and down41 stream region create a complex region in which several 42 magnetospheric phenomena of diverse nature have been 43 observed, like Short Large Amplitude Magnetic Struc44 tures (SLAMS), reconnecting current sheets and fast 45 plasma flows [5-7]. One important property of quasi46 parallel shocks is the formation of jets downstream of a 47 shock with high dynamic pressure, well above the solar 48 wind dynamic pressure [10-12]. They have, e.g., been 49 suggested to trigger magnetopause reconnection [13], ex50 cite surface eigenmodes on the magnetopause [14] and ac51 celerate electrons [15]. Some proposed generation mech52 anisms connect jets to the solar wind interaction with

* Corresponding author: savvra@kth.se
53 the local inclination of bow shock ripples $[12,16,17]$ 54 or to solar wind discontinuities [18]. Although several 55 mechanisms have been proposed to explain how jets are 56 generated, their origin is still not understood.

Here we use data from recently available unique ${ }_{58}$ "string-of-pearls" configuration of the four MMS space59 craft [19] that for the first time allow to follow the ${ }_{60}$ jet formation at the shock. In contrast to earlier sug61 gested mechanisms, we show that super-magnetosonic ${ }_{62}$ jets downstream of the quasi-parallel bow shock can be 63 generated as a direct consequence of the upstream wave ${ }_{64}$ evolution and the bow shock reformation cycle. This ${ }_{65}$ is the first time in-situ observations provide clear evi${ }_{66}$ dence of jet generation as a direct result of the dynam${ }_{67}$ ical evolution of the bow shock. This is fundamentally 68 different compared to other proposed mechanisms that 69 require the presence of external factors (e.g., discontinu70 ities [18]) or specific geometric configurations (e.g., rip71 ples [16]) to take place to explain jets' generation. Fur72 thermore, we observe localized downstream density en73 hancements ("embedded plasmoids" [17, 20]) generated 74 by the same process. The "string-of-pearls" configura75 tion and the relatively stable shock conditions allow us 76 to observe the evolution of both phenomena, originating 77 at the upstream region, evolving and ending up down78 stream in the magnetosheath.

\section{9 \\ Results}

80 Overview. We use data from the MMS spacecraft ${ }_{81}[19]$ on 2019-02-12 from 14:56:50 UTC to 14:58:20 UTC. ${ }_{82}$ FIG. 1(a) shows the satellite separation in the $x y$ and ${ }_{83} x z$ plane, while FIG. 1 (b-e) and FIG. 2 provide ion and 84 magnetic field measurements for the corresponding pe${ }_{85}$ riod. The spacecraft are presented from the farthermost 86 (MMS2) to the closest (MMS3) with respect to Earth. ${ }_{87}$ Starting at 14:57:06, MMS2 observes a localized struc88 ture of increased magnetic field and density (red shaded 89 region, numbered "1"). The magnetic structure is left90 hand polarized (spacecraft reference frame) and as dis91 cussed below is travelling towards the Earth. These prop92 erties along with the localized increase in $|B|$ and den93 sity correspond to typical properties of a SLAMS. As 94 observed by MMS2, this structure is initially upstream 95 from the Earth's bow shock, while from the point of view 
(b)

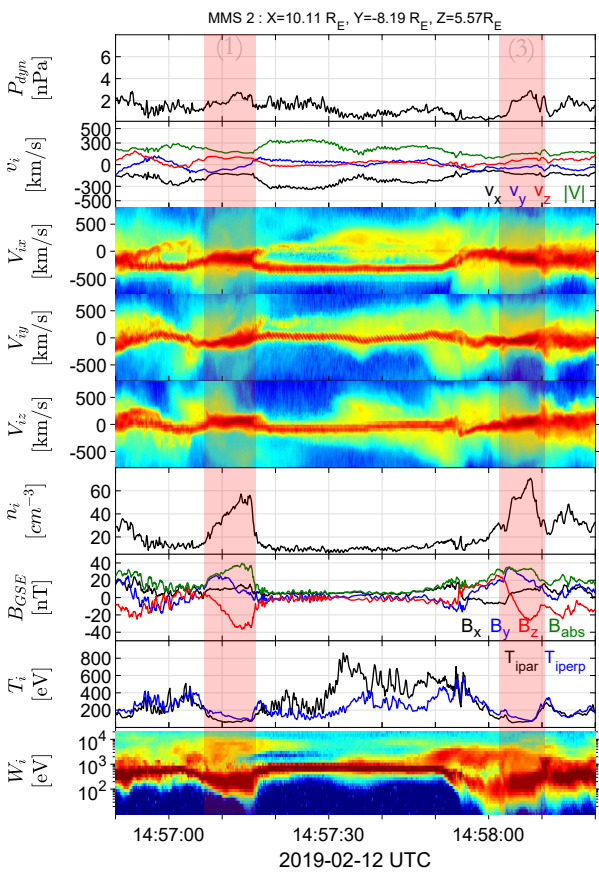

$\nabla$

(d)

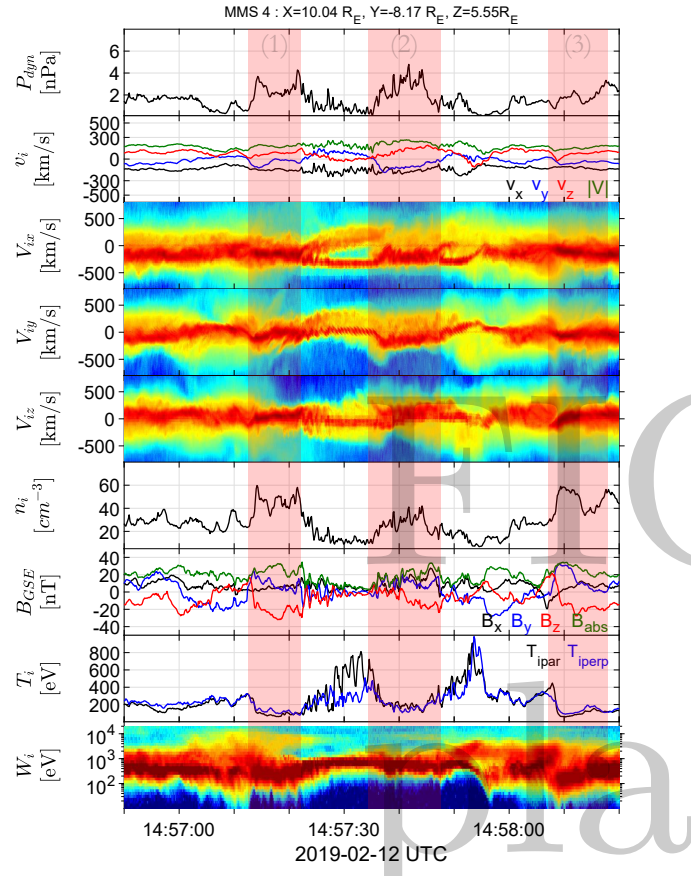

口MMS1

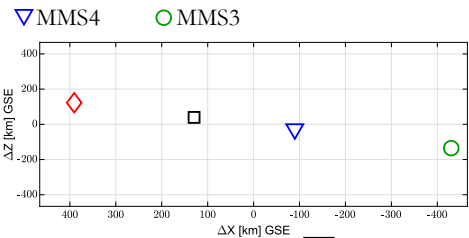

(c)

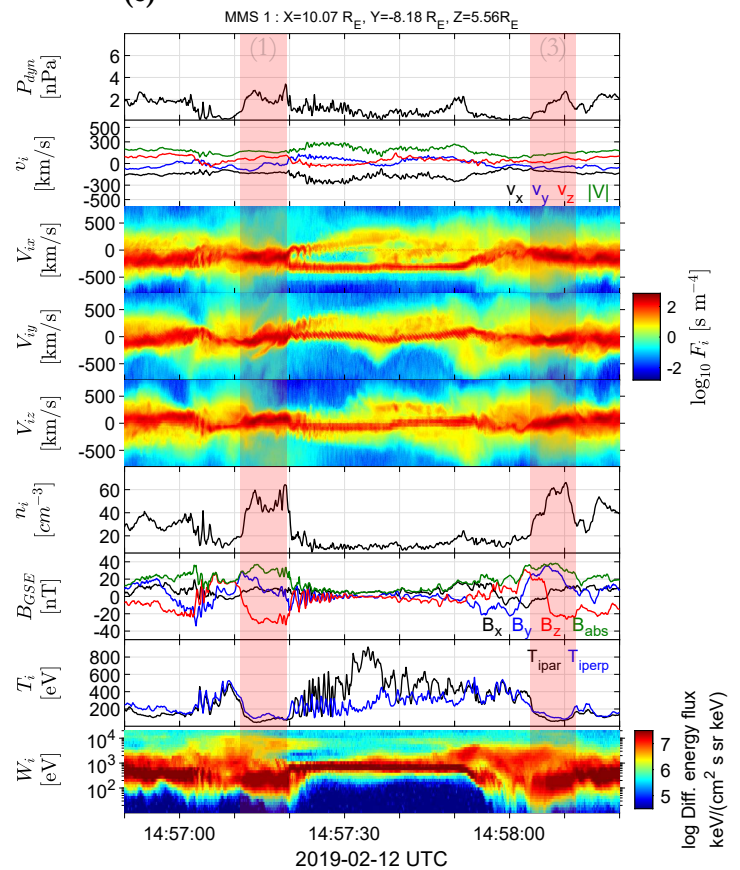

(e)

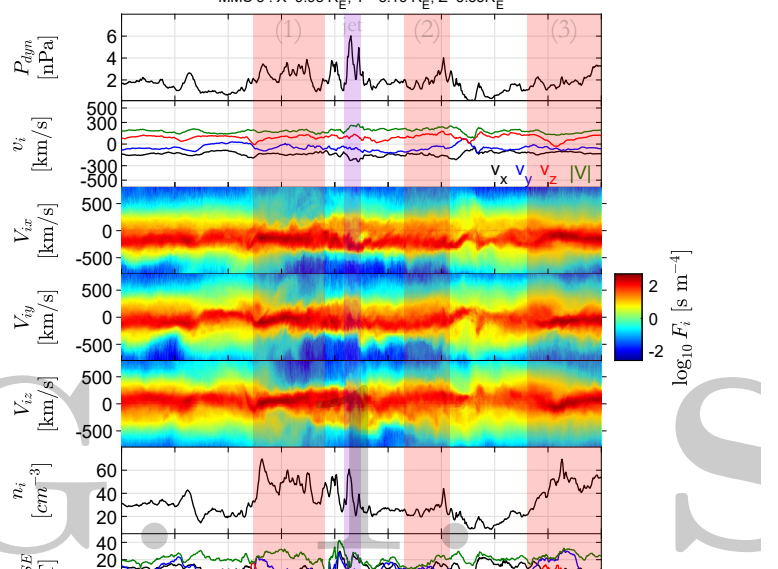

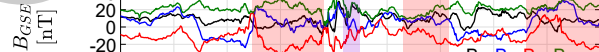

贫

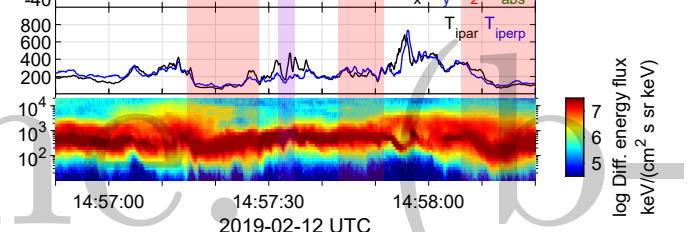



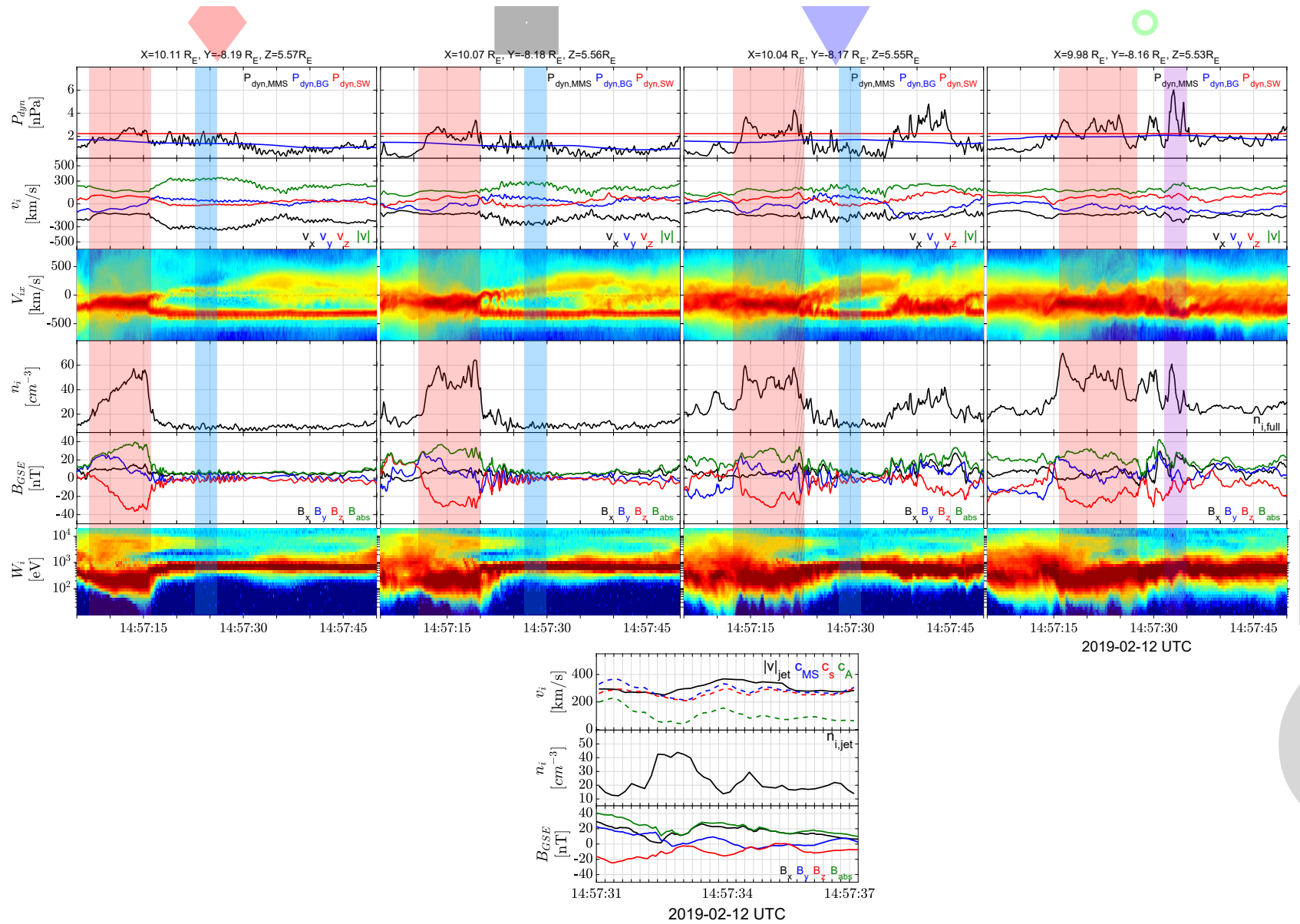
132 ulations [22, 23] and other observational studies [21, 24]. ${ }_{133}$ Moving downstream of the shock, as observed by MMS3, 134 the evolved structure ("1") appears as a "shock rem135 nant" of enhanced density and magnetic field located in 136 the magnetosheath region. The structure now includes a ${ }_{137}$ pile-up region (patterned red region) of the waves associ${ }_{138}$ ated to the shock's ramp evolution. Similar events have 139 been discussed in recent studies $[17,25]$

${ }_{140}$ At approximately 14:57:35 - 14:57:45 (as viewed in ${ }_{141}$ FIG. 3), MMS4 observes a new compressed plasma region 142 ("2"), spatially detached from the initial shock ("1"), ${ }_{143}$ forming upstream of the first, becoming the new local ${ }_{144}$ shock front, and thus completing a bow shock reforma145 tion process/cycle. Returning to the global picture shown 146 in FIG. 1, one can now note that this reformation pro147 cess arise again with the appearance of region "3". As 148 a result, locally, the outer edge of the shock change, fol149 lowing the numbered shaded regions "1", "2" and finally ${ }_{150}$ "3". This process can explain the different observations 151 made by the two outer spacecraft (MMS 1-2) and the 152 inner ones (MMS 3-4). This process has been hypoth153 esized and reported in simulations of the quasi-parallel ${ }_{154}$ shock [26-29].

155 Jet observations. Having established the different 156 regions and the shock reformation process, we proceed to ${ }_{157}$ interpret the super-magnetosonic jet observations made 158 by MMS3. For this observation, an explanation is re159 quired for the enhanced bulk ion velocity and density of 160 the jet. The full particle moments (FIG. 1 and FIG. 2) ${ }_{161}$ show that inside the jet $|V| \approx 220 \mathrm{~km} / \mathrm{s}$ and $n \sim 60$ ${ }_{162} \mathrm{~cm}^{-3}$. The jet however contains two different ion pop${ }_{163}$ ulations, a background magnetosheath and a beam-like 164 jet. Calculating the moments for the beam-like part of 165 the distribution, we obtain $|V| \sim 350 \mathrm{~km} / \mathrm{s}$ and $n \sim 40$ ${ }_{166} \mathrm{~cm}^{-3}$ (see FIG. 2(e) and FIG. S1). This corresponds 167 to a relative increase of $\sim 200 \%$ in dynamic pressure 168 compared to both background magnetosheath and so${ }_{169}$ lar wind levels. Thus, the beam-like jet population has 170 higher density but very similar velocity and temperature 171 to the solar wind observed by the other MMS spacecraft 172 upstream of the shock. The observed increase in density 173 can be explained by a gyro-trapping mechanism originat174 ing from the evolution of the whistler waves upstream of 175 structure "1". Ion trapping between solitary magnetic 176 structures has been recently reported ([21, 24]), seen as 177 an anti-correlation between the plasma density and mag178 netic field magnitude. Here, we observe the same sig179 natures. Finally, the enhanced velocity of the jet rel180 atively to the magnetosheath can be explained by the ${ }_{181}$ effect of the reformation cycle. The beam-like jet pop182 ulation, found within the evolving upstream waves, is 183 effectively transferred downstream of the shock having 184 little to no interaction with the shock environment im185 posed by the initial SLAMS ("1"). Through the refor186 mation process, a new shock front forms upstream of the ${ }_{187}$ jet enclosing it with thermalized magnetosheath plasma 188 and thus completing its formation.

Jet generation and reformation process. Com-

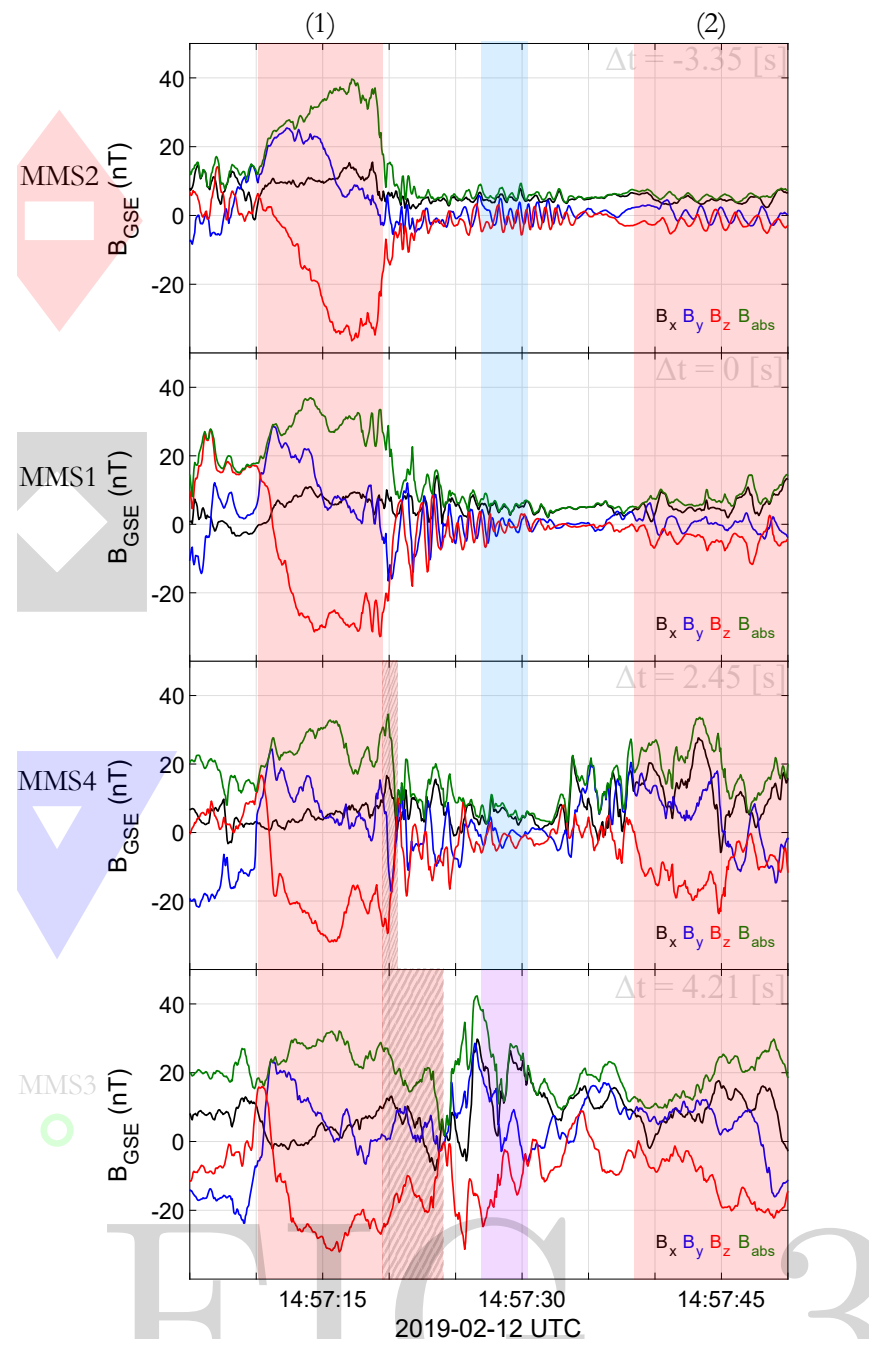




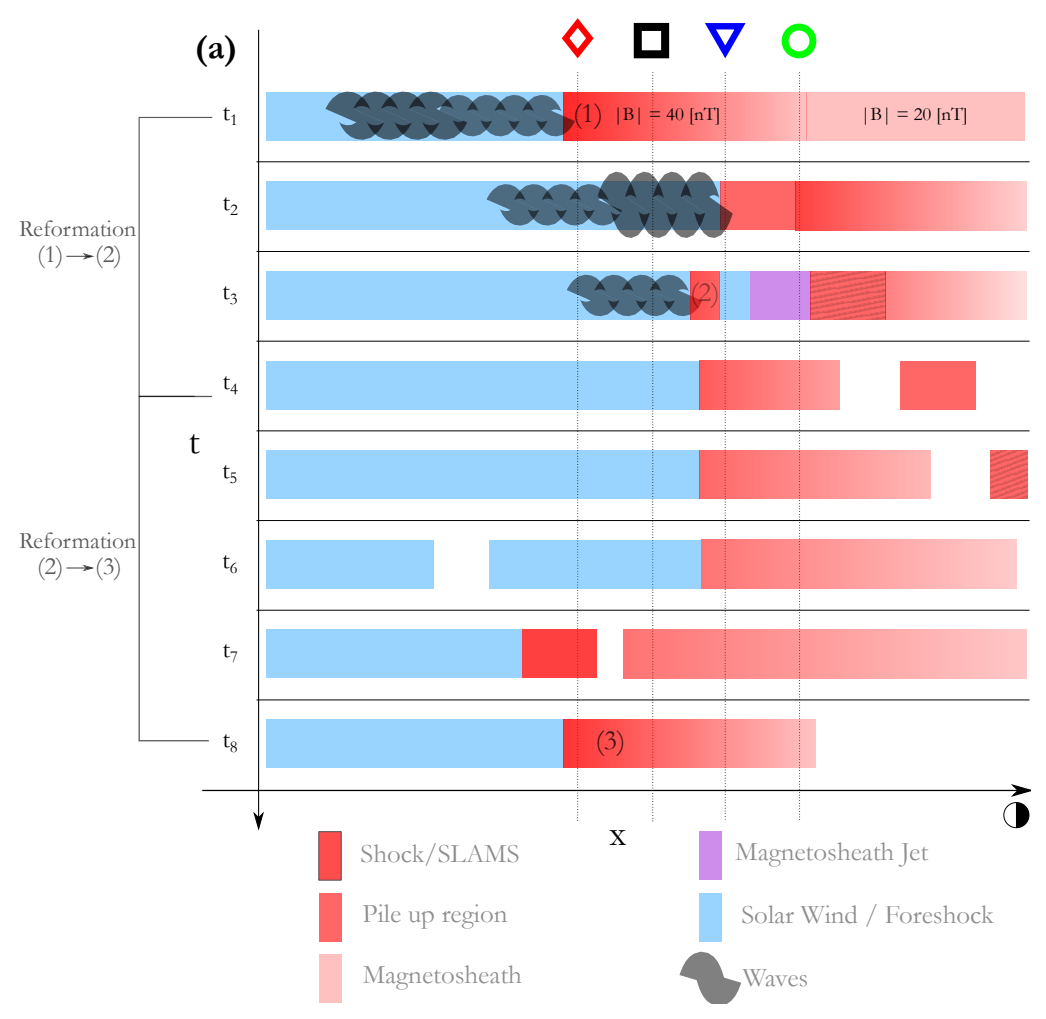

(b)
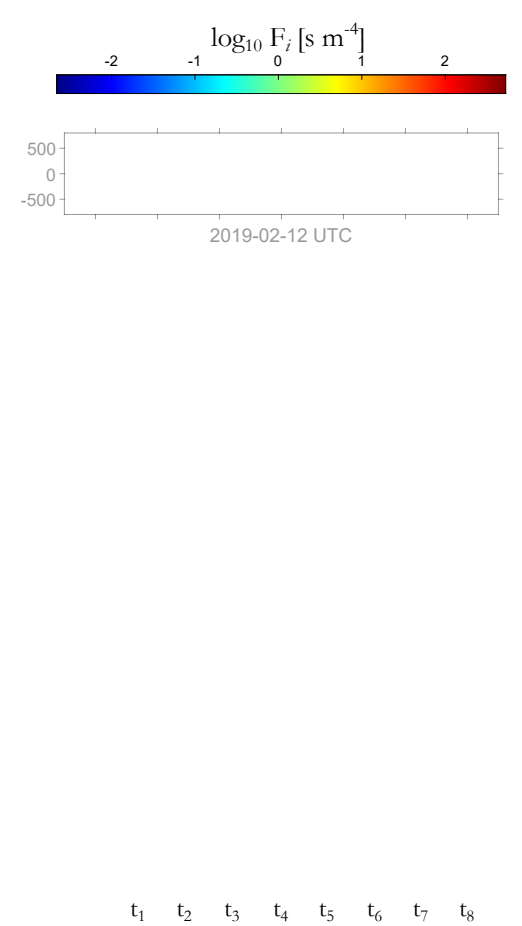
245 important to near-Earth space but also to planetary and ${ }_{289}$ (region "1"). The required time shift is obtained from 246 astrophysical plasmas where collisionless shocks are ubiq- 290 the maximum sample cross-correlation peak lag [34]. The ${ }_{247}$ uitous. The results of this work are a direct success of the 291 maximum correlations between the signals for the time ${ }_{248}$ MMS mission that with its state-of-the-art instruments 292 lags used are $\rho_{1,2}=0.87, \rho_{3,2}=0.44, \rho_{4,2}=0.78$. More 249 and the "string-of-pearls" configuration has enabled the 293 details can be found in [35].

250 discovery of the new jet formation mechanism. Specif- 294 MVA and distribution functions. The polariza251 ically, this discovery is of high importance for design- 295 tion of the SLAMS (structure "1") is obtained with $252 \mathrm{ing}$ and operating future spacecraft missions studying the 296 the help of the minimum-variance analysis (MVA) ${ }_{253}$ bow shock. Further work could address in detail the ex- 297 [36]. Partial ion moments of the jet-like population of ${ }_{254}$ act properties of the ion trapping mechanism and the cor- 298 the magnetosheath jet are computed based on the 2D 255 responding role of electron dynamics. More importantly, 299 reduced distribution functions where the specific ion 256 it is still unknown if similar mechanisms can produce 300 population can be clearly seen at negative $V_{x}$ and $V_{y}$, ${ }_{257}$ more extended flows downstream of the bow shock. Fi- 301 see also supplementary FIG S1. The implementation 258 nally, a statistical analysis and a comparison with global 302 of the minimum-variance analysis, the reduction of 259 simulations is the next logical step.

260

\section{Method}

261 Data. In this work, data from the MMS mission ${ }_{262}$ [19] are primarily used, while for the estimation of up${ }_{263}$ stream solar wind dynamic pressure, we use the OMNI${ }_{264}$ Web database [30]. The magnetic field measurements of 265 MMS are from the fluxgate magnetometer (FGM) [31] of 266 the FIELDS instrument suite [32], sampled every 0.0625 ${ }_{267} \mathrm{~S}$. The fast plasma investigation (FPI) instrument [33] ${ }_{268}$ gives distribution function measurements with a cadence ${ }_{269} 0.15 \mathrm{~s}$ for ions and $0.03 \mathrm{~s}$ for electrons. The solar wind dy270 namic pressure and the model location of the bow shock 271 and magnetopause positions are taken from the OMNI${ }_{272}$ Web dataset [30]. All the vector quantities are in Geo273 centric Solar Ecliptic (GSE) coordinates.

${ }_{274}$ Characteristic velocities. For FIG. 1 and FIG. 2, 275 the dynamic pressure is calculated as: $P_{i, d y n}=\rho_{i} \cdot|V|_{i}^{2}=$ ${ }_{276} n_{i} \cdot m_{p} \cdot|V|_{i}^{2}$. The background magnetosheath dynamic ${ }_{277}$ pressure is calculated by using a moving average window 278 of 300 seconds. For FIG. 4(e), the sound speed is es279 timated as $c_{s}=\sqrt{\left(K T_{e}+\gamma_{i} K T_{i}\right) / M}$, where $K$ is the 280 Boltzmann constant, $T_{i, e}$ is the ion and electron temper281 ature, $M$ is the proton mass and $\gamma_{i}=3$. The Alfvén 282 velocity is calculated as $c_{A}=|B| / \sqrt{\mu_{0} \cdot \rho_{i}}$, where $\rho_{i}$ is 283 the ion mass density, and the magnetosonic velocity is ${ }_{284}$ computed as $c_{M S}=\sqrt{c_{s}^{2}+c_{A}^{2}}$.

285 Cross-correlation and timing. For FIG.3, MMS1 is 286 used as a reference spacecraft to time-shift the measure${ }_{287}$ ments of the other spacecraft so that the time-series rep${ }_{288}$ resent a co-moving view from the initial shock/SLAMS
303 the VDFs to $2 \mathrm{D}$ and $1 \mathrm{D}$ and the computation of the 304 partial moments based on specific parts of the re305 duced 2D VDFs is done via the functions of irfu-matlab 306 package, openly available at https://github.com/irfu. 307 308 Acknowledgments

We thank the MMS team for providing data and sup310 port. We acknowledge the use of NASA/GSFC's Space 311 Physics Data Facility's OMNIWeb service, and OMNI 312 data. We acknowledge the use of irfu-matlab package, 313 https://github.com/irfu. We thank M. Lindberg and 314 A. Lalti, for their comments on the initial stage of the 315 work. We are also thankful for the useful discussions done 316 with the International Space Sciences Institute (ISSI) 317 team, "Foreshocks Across The Heliosphere: System Spe318 cific Or Universal Physical Processes?"

SR and TK are supported by the Swedish National 320 Space Agency (SNSA grant 90/17). FP is supported by ${ }_{321}$ the Austrian Science Fund (FWF): P 33285-N

322 ${ }_{323}$ Data availability

324 Magnetospheric Multiscale (MMS) are available 325 through https://lasp.colorado.edu/mms/sdc/ 326 public/ and OMNI high-resolution data are available 327 through https://omniweb.gsfc.nasa.gov/form/ 328 omni_min.html.

\section{9}

330 Author contributions

S.R. Performed the data analysis and wrote the 332 manuscript. T.K and A.V. supervised the study and con333 tributed to parts of the manuscript through reviews and 334 edits. C.P., F.P., A.J., H.T., and P-A.L. contributed to 335 writing of the manuscripts through reviews and edits. All 336 authors contributed to the interpretation and the discus337 sion of the results.
[1] R. Treumann, Fundamentals of collisionless shocks for 344 astrophysical application, 1. non-relativistic shocks, The 345 Astronomy and Astrophysics Review 17, 409 (2009). 346

[2] A. Balogh and R. A. Treumann, Physics of Collisionless 347 Shocks: Space plasma shock waves (Springer Science \& 348 Business Media, 2013).
[3] D. Caprioli and A. Spitkovsky, Simulations of ion acceleration at non-relativistic shocks. i. acceleration efficiency, The Astrophysical Journal 783, 91 (2014).

[4] A. Bell, Cosmic ray acceleration, Astroparticle Physics 43, 56 (2013).

[5] D. Burgess, E. Lucek, M. Scholer, S. Bale, M. Balikhin, 
A. Balogh, T. Horbury, V. Krasnoselskikh, H. Kucharek, 414 B. Lembège, et al., Quasi-parallel shock structure and 415 processes, Space science reviews 118, 205 (2005).

6] E. Lucek, D. Constantinescu, M. Goldstein, J. Pickett, ${ }_{417}$ J. Pinçon, F. Sahraoui, R. Treumann, and S. Walker, The ${ }_{418}$ magnetosheath, Space Science Reviews 118, 95 (2005). 419

[7] A. Retinò, D. Sundkvist, A. Vaivads, F. Mozer, 420 M. André, and C. Owen, In situ evidence of magnetic ${ }_{421}$ reconnection in turbulent plasma, Nature Physics 3, 235 (2007).

[8] A. Masters, L. Stawarz, M. Fujimoto, S. J. Schwartz, 424 N. Sergis, M. F. Thomsen, A. Retino, H. Hasegawa, 425 B. Zieger, G. R. Lewis, et al., Electron acceleration to 426 relativistic energies at a strong quasi-parallel shock wave, ${ }_{427}$ Nature Physics 9, 164 (2013).

[9] F. Fiuza, G. Swadling, A. Grassi, H. Rinderknecht, ${ }_{429}$ D. Higginson, D. Ryutov, C. Bruulsema, R. Drake, 430 S. Funk, S. Glenzer, et al., Electron acceleration in ${ }^{431}$ laboratory-produced turbulent collisionless shocks, Na- ${ }^{432}$ ture Physics 16, 916 (2020).

10] Z. Němeček, J. Šafránková, L. Přech, D. G. Sibeck, 434 S. Kokubun, and T. Mukai, Transient flux enhancements 435 in the magnetosheath, Geophysical Research Letters 25, 436 1273 (1998). A. Vaivads, M. Palmroth, T. Pulkkinen, H. E. Koskinen, 439 E. Lucek, and H. Rème, Supermagnetosonic jets behind a 440 collisionless quasiparallel shock, Physical Review Letters ${ }_{441}$ 103, 245001 (2009).

[12] F. Plaschke, H. Hietala, M. Archer, X. Blanco-Cano, 443 P. Kajdič, T. Karlsson, S. H. Lee, N. Omidi, M. Palm- 444 roth, V. Roytershteyn, et al., Jets downstream of colli- ${ }_{445}$ sionless shocks, Space Science Reviews 214, 1 (2018). $\quad 446$

[13] H. Hietala, T. D. Phan, V. Angelopoulos, M. Oieroset, 447 M. O. Archer, T. Karlsson, and F. Plaschke, In situ ob- 448 servations of a magnetosheath high-speed jet triggering 449 magnetopause reconnection, Geophysical Research Letters $\mathbf{4 5}, 1732$ (2018).

14] M. Archer, H. Hietala, M. Hartinger, F. Plaschke, and ${ }_{452}$ V. Angelopoulos, Direct observations of a surface eigen- 453 mode of the dayside magnetopause, Nature Communica- 454 tions 10 (2019).

[15] T. Z. Liu, H. Hietala, V. Angelopoulos, R. Vainio, 456 and Y. Omelchenko, Electron acceleration by magne- 457 tosheath jet-driven bow waves, Journal of Geophysical 458 Research: Space Physics 125, e2019JA027709 (2020), 459 e2019JA027709 2019JA027709.

16] H. Hietala and F. Plaschke, On the generation of magne- 46 tosheath high-speed jets by bow shock ripples, Journal of ${ }_{462}$ Geophysical Research: Space Physics 118, 7237 (2013). ${ }_{463}$

17] L. Preisser, X. Blanco-Cano, P. Kajdič, D. Burgess, and ${ }_{464}$ D. Trotta, Magnetosheath jets and plasmoids: Charac- 465 teristics and formation mechanisms from hybrid simula- 460 tions, The Astrophysical Journal 900, L6 (2020).

[18] M. O. Archer, T. S. Horbury, and J. P. Eastwood, Mag- ${ }_{468}$ netosheath pressure pulses: Generation downstream of 469 the bow shock from solar wind discontinuities, Journal 470 of Geophysical Research: Space Physics 117 (2012).

[19] J. Burch, T. Moore, R. Torbert, and B. Giles, Magneto- 472 spheric multiscale overview and science objectives, Space 473 Science Reviews 199, 5 (2016).

20] T Karlsson, A Kullen, E Liljeblad, N Brenning, 4t H. Nilsson, H. Gunell, and M. Hamrin, On the origin 476 of magnetosheath plasmoids and their relation to mag- 477 netosheath jets, Journal of Geophysical Research: Space Physics 120, 7390 (2015).

[21] D. L. Turner, L. B. Wilson, K. A. Goodrich, H. Madanian, S. J. Schwartz, T. Z. Liu, A. Johlander, D. Caprioli, I. J. Cohen, D. Gershman, H. Hietala, J. H. Westlake, B. Lavraud, O. L. Contel, and J. L. Burch, Direct multipoint observations capturing the reformation of a supercritical fast magnetosonic shock, The Astrophysical Journal Letters 911, L31 (2021).

[22] M. Scholer, H. Kucharek, and I. Shinohara, Short largeamplitude magnetic structures and whistler wave precursors in a full-particle quasi-parallel shock simulation, Journal of Geophysical Research: Space Physics 108 (2003).

[23] K. Tsubouchi and B. Lembège, Full particle simulations of short large-amplitude magnetic structures (slams) in quasi-parallel shocks, Journal of Geophysical Research: Space Physics 109 (2004).

[24] L.-J. Chen, S. Wang, J. Ng, N. Bessho, J.-M. Tang, S. F. Fung, G. Le, D. Gershman, B. Giles, C. T. Russell, R. Torbert, and J. Burch, Solitary magnetic structures at quasi-parallel collisionless shocks: Formation, Geophysical Research Letters 48, e2020GL090800 (2021), e2020GL090800 2020GL090800.

[25] T. Z. Liu, Y. Hao, L. B. Wilson III, D. L. Turner, and H. Zhang, Magnetospheric multiscale observations of earth's oblique bow shock reformation by foreshock ultralow-frequency waves, Geophysical Research Letters 48, e2020GL091184 (2021), e2020GL091184 2020GL091184.

[26] S. J. Schwartz, M. F. Thomsen, and J. T. Gosling, Ions upstream of the earth's bow shock: A theoretical comparison of alternative source populations, Journal of Geophysical Research: Space Physics 88, 2039 (1983).

[27] D. Burgess, Cyclic behavior at quasi-parallel collisionless shocks, Geophysical research letters 16, 345 (1989).

[28] Y. Lin and X. Y. Wang, Three-dimensional global hybrid simulation of dayside dynamics associated with the quasiparallel bow shock, Journal of Geophysical Research: Space Physics 110 (2005).

[29] Y. Hao, X. Gao, Q. Lu, C. Huang, R. Wang, and S. Wang, Reformation of rippled quasi-parallel shocks: 2-d hybrid simulations, Journal of Geophysical Research: Space Physics 122, 6385 (2017).

[30] J. King and N. Papitashvili, Solar wind spatial scales in and comparisons of hourly wind and ace plasma and magnetic field data, Journal of Geophysical Research: Space Physics 110 (2005).

[31] C. Russell, B. Anderson, W. Baumjohann, K. Bromund, D. Dearborn, D. Fischer, G. Le, H. Leinweber, D. Leneman, W. Magnes, et al., The magnetospheric multiscale magnetometers, Space Science Reviews 199, 189 (2016).

[32] R. Torbert, C. Russell, W. Magnes, R. Ergun, P.-A. Lindqvist, O. LeContel, H. Vaith, J. Macri, S. Myers, D. Rau, et al., The fields instrument suite on mms: Scientific objectives, measurements, and data products, Space Science Reviews 199, 105 (2016).

[33] C. Pollock, T. Moore, A. Jacques, J. Burch, U. Gliese, Y. Saito, T. Omoto, L. Avanov, A. Barrie, V. Coffey, et al., Fast plasma investigation for magnetospheric multiscale, Space Science Reviews 199, 331 (2016).

[34] G. E. Box, G. M. Jenkins, G. C. Reinsel, and G. M. Ljung, Time series analysis: forecasting and control (John Wiley \& Sons, 2015). 
478 [35] G. Paschmann and P. W. Daly, Analysis methods for ${ }_{482}$ [36] 479 multi-spacecraft data. issi scientific reports series sr-001, 483 esa/issi, vol. 1. isbn 1608-280x, 1998, ISSI Scientific Re- 484 ports Series 1 (1998).
[36] G. Paschmann and S. Schwartz, Issi book on analysis methods for multi-spacecraft data, in Cluster-II workshop multiscale/multipoint plasma measurements, Vol. 449 (2000) p. 99. 


\section{Supplementary Files}

This is a list of supplementary files associated with this preprint. Click to download.

- supportinginformation.pdf 